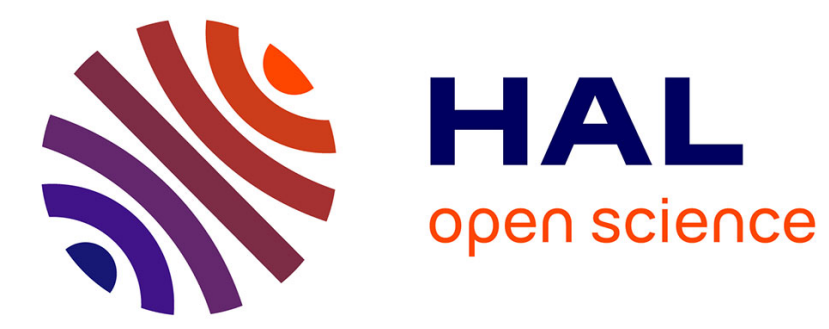

\title{
Les Lumières de Charles Taylor
}

Céline Spector

\section{To cite this version:}

Céline Spector. Les Lumières de Charles Taylor. Revue de Métaphysique et de Morale, 2020, Charles

Taylor, 108, pp.497-512. 10.3917/rmm.204.0497 . hal-03149815

\section{HAL Id: hal-03149815 \\ https: / hal.sorbonne-universite.fr/hal-03149815}

Submitted on 23 Feb 2021

HAL is a multi-disciplinary open access archive for the deposit and dissemination of scientific research documents, whether they are published or not. The documents may come from teaching and research institutions in France or abroad, or from public or private research centers.
L'archive ouverte pluridisciplinaire HAL, est destinée au dépôt et à la diffusion de documents scientifiques de niveau recherche, publiés ou non, émanant des établissements d'enseignement et de recherche français ou étrangers, des laboratoires publics ou privés. 


\section{Les Lumières de Charles Taylor}

RÉSUMÉ. Cette contribution tentera de cerner les enjeux de l'interprétation contrastée de la philosophie des Lumières proposée par Charles Taylor. Pourquoi le déisme et le matérialisme sont-ils perçus par Taylor comme des fourvoiements de la modernité ? Pourquoi considère-t-il que les Lumières ont méconnu la manière dont la subjectivité pouvait fonder ses aspirations morales et politiques? Nous verrons que la philosophie des Lumières " radicales ", selon Taylor, produit une morale et une politique incohérentes : elle postule que l'harmonie sociale procède de l'intérêt bien compris grâce à une ingénierie législative et éducative; mais elle n'explique pas pourquoi l'idéal d'une société harmonieuse mérite d'être poursuivi. L'auteur des Sources du moi entérine de la sorte l'idée répandue d'une incohérence des philosophes matérialistes : leur psychologie hédoniste et égoïste est incompatible avec leur éthique, qui vise le plus grand bonheur du plus grand nombre. Les Lumières sont donc rendues responsables d'une vision contradictoire de la morale et d'une vision appauvrie de la subjectivité.

ABSTRACT. This contribution will question Charles Taylor's interpretation of the Enlightenment. Why did he analyze deism and materialism as a misdirection of modernity? Why did he consider that Enlightenment philosophy misunderstood the way in which subjectivity could ground its moral and political aspirations? I will show that the "radical" Enlightenment, according to Taylor, produces an incoherent account of morality and politics: it postulates that social harmony proceeds from self-interest, thanks to legislative and educational engineering; but it does not explain why the ideal of a harmonious society is worth pursuing. The author of The Sources of the Self thus confirms the widespread idea of an incoherence of the materialist philosophes: their hedonistic and selfish psychology is incompatible with their ethics, which aims for the greatest happiness of the greatest number. The Enlightenment is therefore made responsible for a contradictory vision of morality and an impoverished vision of the self.

Selon Charles Taylor, les Lumières sont la source de l'identité moderne et des deux voies, instrumentale et expressiviste, que celle-ci peut emprunter. Dans le chapitre des Sources du moi consacré aux horizons moraux fragmentés de la modernité, le philosophe distingue deux constellations d'idées issues des Lumières : l'une attache la raison "désengagée » à une interprétation instrumentale de la nature ; l'autre ancre l'imagination dans un sentiment conçu comme source morale intérieure. Or « la tension qui s'exerce entre elles constitue l'un des traits dominants de la culture moderne $»^{1}$. C. Taylor retrace d'abord la genèse de la première constellation, soit la mutation par laquelle la pensée des Lumières radicales athées se substitue au déisme anglais chez des auteurs matérialistes ou utilitaristes comme d'Holbach, Helvétius ou Bentham. Mais cette tendance n'est pas seule en lice : la modernité est riche d'une autre généalogie du sujet moral. Tout l'effort de Taylor vise précisément à l'exhumer et à en manifester l'importance: l'idée de "nature-source» ouvre une voie féconde dont le romantisme, héritier de Rousseau, sera l'une des expressions privilégiées.

Cette contribution tentera de cerner les enjeux de cette interprétation contrastée des Lumières - concept que $\mathrm{C}$. Taylor utilise malgré les difficultés méthodologiques qui grèvent

\footnotetext{
${ }^{1}$ C. TAYlor, Les Sources du moi. La formation de l'identité moderne, trad. C. Mélançon, Paris, Seuil, 1998 (désormais $S M$ ), p. 407.
} 
son usage ${ }^{2}$. Pourquoi le déisme et le matérialisme sont-ils perçus par Taylor comme des fourvoiements de la modernité ? Pourquoi considère-t-il que les Lumières ont méconnu la manière dont le sujet devait fonder ses aspirations morales et politiques ? Pour quelles raisons se joint-il aux innombrables réquisitoires versés au procès des Lumières (Strauss, Adorno, Horkheimer, Foucault, MacIntyre et d'autres) ${ }^{3}$ ?

Nous verrons que la philosophie des Lumières « radicales », selon C. Taylor, produit une morale et une politique incohérentes : elle postule que l'harmonie sociale procède de l'intérêt bien compris grâce à une ingénierie législative et éducative ; mais elle n'explique pas pourquoi l'idéal d'une société harmonieuse mérite d'être poursuivi. L'auteur des Sources du moi entérine l'idée répandue d'une incohérence des philosophes matérialistes : leur psychologie hédoniste et égoïste serait incompatible avec leur éthique, qui vise le plus grand bonheur du plus grand nombre. Les Lumières sont donc rendues responsables d'une vision contradictoire de la morale et d'une vision appauvrie de la subjectivité. Dès lors, l'espoir ne peut résider que dans la réaction romantique contre le Moi rationaliste et désengagé : grâce à l'imagination créatrice, l'expressivisme serait voué à revitaliser la nature et à rendre un monde signifiant au Moi moral. Notre modernité devrait donc poursuivre cette voie, plutôt que celle des Lumières " radicales », dont les effets pervers se seraient faits connaître lors de la Terreur, voire des totalitarismes.

\section{Les contradictions des Lumières radicales}

La généalogie des Lumières esquissée par Charles Taylor fait de la philosophie déiste et des théories du «sens moral» un moment de rupture avec les théories antiques du «bien cosmique » comme avec la vision cartésienne de la morale. Selon le grand récit des Sources $d u$ $M o i$, cette philosophie n'est pas dénuée de danger : en fondant les jugements en matière morale sur des données brutes (les sentiments), Shaftesbury et Hutcheson suscitent une possibilité nouvelle : que l'on puisse considérer les sentiments moraux comme de simples projections de l'esprit. Leurs théories ouvrent la voie au relativisme (Dieu aurait pu nous créer avec d'autres sens) et au naturalisme, si l'on fait l'économie de Dieu ou de la Providence qui "cautionne » le sens moral. L'intériorisation moderne des sources de la morale est indissociable de cette création d'une vulnérabilité théorique : la critique du rationalisme et de l'objectivisme moral s'accompagne d'un risque, celui du «projectivisme» et, ultimement, du relativisme subjectiviste.

Mais le pire reste à venir. Dans la quatrième partie des Sources du moi, C. Taylor s'attache à définir son véritable adversaire : le radicalisme des Lumières, assimilé à un «naturalisme» et à un "utilitarisme ». Les Aufklärer radicaux opèrent en effet la mue du déisme ou du théisme en athéisme. Ayant récusé toute idée de Providence, ils justifient une vision purement immanente de la morale. Si nos jugements sur le bien et le mal ne relèvent ni de l'ancien ordre hiérarchique associé au cosmos, ni de l'ordre moderne de la finalité providentielle, ils ne peuvent porter que sur les conséquences de nos actes. Selon les philosophes, il s'agit d'utiliser les relations causales découvertes dans la nature pour être en mesure de produire la plus grande utilité sociale ou le plus grand bonheur du plus grand nombre. Ceux que Taylor nomme ainsi les « utilitaristes » rejettent le «bien constitutif» du déisme, mais ils conservent les trois idéaux que l'ordre déiste avait étayé : l'idéal de la raison

\footnotetext{
${ }^{2}$ C. TAYlor, «The Immanent Counter-Enlightenment », in R. BeInER, W. Norman (dir.), Canadian Political Philosophy: Contemporary Reflections, Oxford, Oxford University Press, 2001, p. 386-400.

${ }^{3}$ Sur ce procès des Lumières, voir les analyses récentes d'A. LiLTi, L'héritage des Lumières. Ambivalences de la modernité, Paris, Seuil, 2019 ; Stéphanie RozA, La Gauche contre les Lumières ?, Paris, Fayard, 2020.
} 
autonome et la liberté à l'égard de toute autorité ; l'importance décisive de la vie ordinaire (travail, famille, patrie) ; l'idéal d'une bienveillance universelle, impartiale et désintéressée.

Le sens du matérialisme des Lumières, tel qu'on le découvre chez Diderot, Helvétius et d'Holbach notamment, s'éclaire alors : il s'agit d'abord d'un sensualisme et d'un hédonisme affirmé. La critique du péché originel et de l'hypothèse augustinienne conduit à soutenir que la nature est innocente, et non corrompue depuis la Chute. Cette innocence de la nature sert à fonder la morale sur un naturalisme étroit et à juger toute aspiration surnaturelle illusoire et vaine : les philosophes français prônent l'affirmation de la vie ordinaire en dénigrant l'idée de fins «plus hautes ", d'idéaux plus "nobles » que ceux que la nature suggère grâce à la recherche du plaisir physique lui-même. Contre l'ascétisme, il faut rendre ses droits au corps ; contre le spiritualisme, démystifier l'idée de fins morales supérieures aux fins physiques. Les penseurs radicaux des Lumières qui embrassèrent l'athéisme et le matérialisme comme expression ultime de la raison autonome le firent aussi, selon C. Taylor, afin d'être sincères envers l'exigence imposée par la nature. C'est le cas de d'Holbach : son Système de la nature exprime un monisme sans compromis. Ici comme chez Helvétius, la promotion de la vie ordinaire est métamorphosée en « exaltation de la sensualité » ${ }^{4}$.

Ce que C. Taylor perçoit chez Diderot, Helvétius, d'Holbach est une version radicale de «l'utilitarisme », qui est une forme d'hédonisme : aux yeux de la coterie holbachique, le désir est intrinsèquement bon. Il faut savoir satisfaire la nature qui nous enjoint de nous conserver et de nous reproduire. Chez Diderot notamment, la satisfaction sexuelle est érigée en valeur suprême face à la foi chrétienne, associée à une morale répressive. Le code civil et le code religieux sont monstrueux s'ils s'opposent au code de la nature; erreurs et vices ne viennent que de ce que l'on contrarie les inclinations naturellement droites de l'humanité. Corrélativement, le but authentique de la vie morale devient la diminution des souffrances associées à la condition humaine; la véritable vertu est une bienveillance universelle et impartiale, et non la vertu chrétienne, toujours soupçonnée de perversion. Cette bienveillance universelle sera désormais au cœur de la morale des modernes.

Faut-il en conclure que la valorisation de la bienveillance n'est que l'effet du développement de la raison scientifique ? Telle est précisément l'hypothèse que Taylor entend réfuter, visant, par-delà les Lumières, la sociobiologie contemporaine ${ }^{5}$. Loin d'être scientistes, les Aufklärer radicaux étaient mus par des motifs moraux. Aussi défendirent-ils farouchement l'idée d'une harmonie des intérêts, qui devrait s'établir à condition que disparaissent les entraves d'une religion obscurantiste et d'une politique despotique. Selon leur versant le mieux connu et le plus critiqué, les Lumières portaient ainsi l'espoir d'un avenir radieux, d'un progrès indéfini grâce à l'éducation et à la législation. À l'instar de Condorcet, nombreux sont les philosophes à avoir voulu articuler progrès scientifiques et progrès éthiques ${ }^{6}$.

Or Taylor veut montrer qu'il existe une contradiction performative dans le naturalisme des Lumières. Les aspirations des philosophes radicaux, selon lui, ne peuvent pas être énoncées dans les termes qu'autorise leur vision de la nature humaine : leur réductionnisme (du moral au

\footnotetext{
${ }^{4}$ SM, p. 416. Pour une présentation plus générale de la démarche de Taylor, voir R. ABBEY, Charles Taylor, Princeton, Princeton University Press, 2000. Nous nous permettons également de renvoyer à C. SPECTOR, "Charles Taylor, philosophe de la culture ", La Vie des idées, 8 avril 2014. URL : http://www.laviedesidees.fr/Charles-Taylor-philosophe-de-la.html

${ }^{5}$ Voir par exemple Edward O. Wilson, La Sociobiologie, Paris/Monaco, Le Rocher, 1987 ; L'Humaine nature. Essai de sociobiologie, Paris, Stock, 1979.

${ }^{6} S M$, p. 420.
} 
physique, du noble au vil) exclut l'idée même d'« évaluation forte » (strong evaluation), c'està-dire la reconnaissance que certaines de nos fins ne relèvent pas de simples " préférences » et sont incommensurables avec nos autres désirs. Telle serait l'attitude paradoxalement naïve de ceux qui veulent tout démystifier. L'abandon des évaluations fortes conduit à ne pouvoir justifier l'idéal moral de bienveillance universelle : "Cela fait de l'utilitarisme une position intellectuelle bien étrange ${ }^{7}$. D'un côté, il importe de faire le bonheur du plus grand nombre et de diminuer la souffrance; de l'autre, nul ne peut rien dire du contenu réel des principes moraux. D'un côté, il faut démystifier les fausses valeurs de la religion et de la morale conventionnelle; de l'autre, on ne peut leur substituer qu'une éthique réductrice où l'altruisme peine à se justifier. Telle est la source d'une tension irréductible, car Diderot, d'Holbach, Helvétius ou Bentham ont besoin d'un « horizon moral » pour soulager la souffrance, corriger les injustices et lutter pour une "noble cause ». D'où les contradictions inhérentes au matérialisme, dont les réfutations d'Helvétius et de La Mettrie formulées par Diderot sont le symptôme ${ }^{8}$. In fine, l'ontologie réductionniste est inconciliable avec l'élan altruiste. Le naturalisme des Lumières est contradictoire dès lors que la raison désengagée ne suffit pas à fonder un idéal moral. L'idée d'amour de l'humanité demeure injustifiée.

Par une singulière ironie du sort, Sade révèle a contrario l'horizon moral des philosophes, leur humanisme finalement illusoire. Comme dans l'œuvre d'Adorno, d'Horkheimer ou de Foucault ${ }^{9}, 1^{\prime}$ auteur de l'Histoire de Juliette permet selon C. Taylor de cerner le sens authentique de la philosophie des Lumières :

\begin{abstract}
Ce que révèlent, en tant que repoussoir, les vues de Sade, c'est l'arrière-plan habituellement invisible de l'humanisme des Lumières, que j'ai appelé précédemment l'horizon moral de leur pensée. Adopter simplement une certaine forme de matérialisme ne suffit pas pour produire toute l'éthique de la bienveillance utilitariste. On a besoin d'une conception contextuelle de ce qui est digne d'évaluation forte : dans ce cas-ci elle concerne la signification morale du bonheur ordinaire et l'exigence de la bienfaisance universelle. On a alors des raisons de réagir aux hypothèses sur le désir et le bonheur des êtres humains à la manière classique de l'universalisme des Lumières. En soi, le matérialisme ne nous donne pas plus de raison d'aller dans ce sens que d'adopter l'égoïsme sadien, comme les contre-Lumières l'ont faussement prétendu. N'être que matérialiste, c'est avoir une position éthique sous-déterminée ${ }^{10}$.
\end{abstract}

L'humanisme des Lumières exige une « ontologie plus riche de la personne humaine et de la nature $\gg{ }^{11}$. Pour justifier le sacrifice de soi à une cause plus noble, il est impossible de s'en remettre à l'espoir d'une harmonie future des intérêts. La société juste représente un idéal qui commande l'allégeance présente de tout un chacun, alors que l'argument scientifique ne saurait passer du is au ought: la raison est incapable de donner sens à cette évaluation. L'auteur des Sources du moi en conclut qu'une évaluation forte est indispensable.

La généalogie des Lumières radicales esquissée par Taylor est donc profondément différente de celle de Leo Strauss, qui percevait dans les deux dernières vagues de la modernité l'avènement de l'historicisme et du nihilisme ${ }^{12}$; elle diffère tout autant de celle que proposera

\footnotetext{
${ }^{7}$ SM, p. 421.

${ }^{8}$ Diderot, Réfutation d'Helvétius, J. Assezat, M. Tourneux (éds.), in CEuvres complètes de Diderot, Paris, Garnier, 1876, vol. II, p. 245-456.

$9^{9}$ Voir P. SABOT, «Foucault, Sade et les Lumières », Lumières, $2^{\mathrm{e}}$ semestre 2006, 8, « Foucault et les Lumières », F. Brugere, G. Le BlanC, C. SPECTOR, J. TERREL (dir.), p. 141-155.

${ }^{10} S M$, p. 425.

${ }^{11} S M$, p. 425.

${ }^{12}$ L. STRAuss, «Les trois vagues de la modernité », in La Philosophie politique et l'Histoire, trad. fr. O. Seyden, Paris, Librairie Générale Française, 2008,p. 211-235. Voir C. Pelluchon, Leo Strauss. Une autre raison, d'autres Lumières. Essai sur la crise de la rationalité contemporaine, Paris, Vrin, 2005.
} 
Jonathan Israël, faisant l'éloge des Lumières radicales émancipatrices ${ }^{13}$. Si le matérialisme ne donne pas plus de raison d'aller vers l'humanisme que vers le sadisme, le radicalisme des Lumières ne peut être que «parasitaire » : il «parasite ses adversaires pour l'expression de ses propres sources morales ${ }^{14}$. Le parasite va chercher chez ses ennemis ses idéaux moraux; il puise ses ressources théoriques dans la culture morale que ceux-ci ont le mieux explicitée. « Bourrées de contradictions », les « Lumières utilitaristes » n'ont de sens qu'en raison des abus de leur ennemi, "l'infâme $»^{15}$. La séduction des Lumières radicales se nourrit surtout des horreurs de la persécution religieuse et du fanatisme chrétien. C'est ce qui explique que cette pensée, parvenue au faîte de son pouvoir sous la Révolution française, révèle son caractère étroit et menaçant. Étroit, car il faut définir des biens de manière positive, ce à quoi les Lumières révolutionnaires ne parviennent pas réellement ; menaçant, car la seule efficacité instrumentale dans la recherche du bonheur peut conduire à la catastrophe : destruction des mœurs et coutumes anciennes, nivellement, élimination sans vergogne de tous les opposants. C. Taylor reprend ici à son compte la critique classique de la Terreur. Comme la bonté morale ne peut être fondée par le radicalisme des Lumières, le risque est que l'informulé (ce bien mal fondé) " reste à demi réprimé », ce qui donne alors au négatif une puissance décisive : " Au bout du compte, l'élan des Lumières peut tourner à une entreprise purement cynique de dénonciation ; l'élan révolutionnaire se définit alors par-dessus tout par l'aspiration à détruire l'ordre établi ${ }^{16}$.

\section{La nature-source}

Il reste néanmoins une planche de salut dans la philosophie des Lumières, du côté de la critique du matérialisme : C. Taylor fait de Rousseau l'origine d'une autre voie empruntée par la formation de l'identité moderne. En premier lieu, la pensée rousseauiste joue un rôle crucial dans l'élaboration de l'idéal moral d'authenticité. L'auteur des Sources du moi est persuadé que cet idéal, dont on dénonce souvent les perversions contemporaines (relativisme, narcissisme, hédonisme), est doté d'une véritable force morale. Or Rousseau est l'un des premiers à lui avoir donné droit de cité : au-delà de l'affirmation selon laquelle la nature humaine est naturellement bonne, l'auteur de l'Émile invoque la conscience comme source d'un jugement infaillible sur le bien et le mal. La voix de la conscience est la voix de la nature qui s'exprime en l'homme. Désormais, la morale n'est plus tributaire de normes transcendantes, issues de Dieu ou d'une tradition extérieure à l'humanité : la moralité consiste à " suivre la voix de la nature » qui est en nous. Si cette voix est souvent étouffée par les passions induites par notre dépendance à l'égard d'autrui (la principale étant l'amour-propre), le salut moral procède de la possibilité de retrouver un lien authentique à soi. Autant que la conscience, que Rousseau définit dans la Profession de foi du Vicaire Savoyard comme un instinct moral, juge infaillible du bien et du mal, Taylor invoque le "sentiment de l'existence » qui témoignerait dans les Rêveries de ce contact intime avec soi, source de plénitude ${ }^{17}$.

Dans Les Sources du moi, Rousseau se trouve de la sorte inclus parmi les sources naturalistes de la morale qui s'épanouissent à partir du XVIII ${ }^{\mathrm{e}}$ siècle. Corrélativement, l'auteur du Contrat social et de l'Émile apparaît, en politique comme en morale, comme un philosophe de la liberté. Il participe à l'invention moderne de l'autonomie :

\footnotetext{
${ }^{13}$ J. ISRAËL fait l'éloge de ce courant qui permit selon lui de penser, à partir de sources spinozistes, l'émancipation des hommes, des femmes, et des esclaves (A Revolution of the Mind, Radical Enlightenment and Intellectual Origins of Modern Democracy, Princeton, Princeton University Press, 2010).

${ }^{14} S M$, p. 429.

${ }^{15}$ SM, p. 429. Voir récemment B. BINOCHE, «Écrasez l'infâme ! », Paris, La Fabrique, 2018.

${ }^{16} S M$, p. 434.

${ }^{17}$ Charles TAYLOR, «Quiproquos et malentendus : le débat communautariens-libéraux », in A. BERTEN, P. DA Silveira, H. Pourtois (dir.), Libéraux et Communautariens, Paris, Puf, 1997, p. 46.
} 
Rousseau se situe à l'origine d'une grande part de la culture contemporaine, autant des philosophies de l'exploration de soi que des credo qui ont fait de la liberté autodéterminée la clé de la vertu. Il est le point de départ d'une transformation dans la culture moderne qui tend vers une intériorité plus profonde et une autonomie radicale. Tous les courants partent de lui ${ }^{18}$.

Enfin, Taylor se tourne vers deux prolongements de la pensée rousseauiste: le formalisme moral kantien, l'expressivisme herderien. Ces deux réactions aux insuffisances du naturalisme des Lumières convergent sur un point au moins : le constat kantien et romantique de la «superficialité » des Lumières. Les deux réactions sont des intériorisations ; toutes deux réagissent à l'absence d'une dimension morale spécifique dans le déisme et le naturalisme classiques. Toutes deux s'insurgent contre une vision appauvrie et " unidimensionnelle » de l'homme ${ }^{19}$.

\section{Une lecture hégélienne des Lumières}

On peut à bon droit s'interroger sur le sens philosophique d'une telle critique. Pourquoi abonder le procès des Lumières matérialistes ? Le réquisitoire de Charles Taylor diffère en profondeur de celui qu'avaient conduit Horkheimer et Adorno dans la Dialectique de l'Aufklärung (1947), mais également de la critique de l'humanisme des Lumières que l'on trouve à l'œuvre chez Foucault pendant la période de Surveiller et Punir $(1975)^{20}$. Elle s'inspire dans une certaine mesure de la lecture d'Isaiah Berlin, le mentor de Taylor à Oxford dans ses jeunes années. Il faut donc cerner la spécificité de cette lecture ambivalente des Lumières, qui entend maintenir vivaces les grands idéaux dont celles-ci seraient porteuses (autonomie, égale dignité, authenticité) tout en critiquant leur naturalisme athée.

C. Taylor prend pour point de départ la critique hégélienne des Lumières, qui auraient défendu une vision utilitaire de la morale et une conception instrumentale du social ${ }^{21}$. Il hérite notamment de la critique adressée par l'auteur de la Phénoménologie de l'esprit au Neveu de Rameau comme symbole des Lumières françaises. À la suite de Goethe et de Schiller, Hegel avait défendu l'œuvre de Diderot contre la réaction romantique associée au nationalisme germanique. À ses yeux, le Neveu incarne face au philosophe un Aufklärer avisé, exercé à la critique réflexive, qui pourchasse les préjugés et les superstitions ${ }^{22}$. En mettant en lumière le rôle de la subjectivité dans la position des valeurs, l'objecteur au philosophe (« Moi ») dissout la confiance dans l'objectivité des normes. Il induit la confusion dans la conscience commune qui comprend les valeurs comme des universaux abstraits. La « conscience vile » provoque la « conscience noble » en mimant la comédie humaine, corrompue par la flatterie mondaine et la domination de l'argent. Mais le Neveu incarne aussi les limites, pour Hegel, des Lumières françaises : celles-ci ne parviennent pas à donner suite à leur esprit critique. La question est de

\footnotetext{
${ }^{18} S M$, p. 455. Sur ce point que nous ne pouvons développer ici, nous nous permettons de renvoyer à C. SPECTOR, «De Rousseau à Charles Taylor. Autonomie, authenticité, reconnaissance », B. BERnARDI, F. GUENARD, B. Bachofen, A. CharraK (dir.), Philosophie de Rousseau, Paris, Classiques Garnier, 2014, p. 349-361.

${ }^{19}$ SM, p. 479.

${ }^{20} \mathrm{C}$. Taylor critique le nietzschéisme foucaldien, qui procède d'une critique des Lumières ignorant le tribut (l'immanentisme) qu'il leur doit (C. TAYLOR, « The Immanent Counter-Enlightenment », art. cit., p. 399).

${ }^{21}$ C. TAYLOR, Hegel et la société moderne, trad. fr. P. Desrosiers, Paris, Cerf, 1998, p. 141-154. Voir J. D'HONDT (dir.), Hegel et le siècle des Lumières, Paris, Puf, 1974 ; J.-F. KERVEGAN, B. MABILlE (dir.), avec la collaboration d'É. Djordjevic, Hegel au présent : une relève de la métaphysique ?, Paris, CNRS Éditions, 2012.

${ }^{22}$ Hegel, Phénoménologie de l'esprit, trad. fr. J.-P. Lefèbvre, Paris, Aubier, 1991, p. 349-356. Voir I. GARO, « Le Neveu de Rameau dans la Phénoménologie de l'Esprit : littérature, droit et philosophie », Europe, oct. 2002,882 ; C. THERIEN, «Les Lumières et la dialectique. De Hegel à Adorno et Horkheimer », Revue Philosophique de Louvain, 2003, 101 (4), p. 568-592.
} 
savoir que faire, une fois l'entendement émancipé de ses tutelles : le Neveu dévoile les effets pervers d'une raison formelle emballée par son pouvoir destructeur, incapable de reconstruire un monde substantiel où l'esprit puisse se retrouver « chez lui ».

C. Taylor s'inspire tout autant de la volonté hégélienne de restaurer une Sittlichkeit plus adaptée aux mœurs depuis l'ère des Révolutions. Associé au moment de l'entendement, l'instrumentalisme étroit des Lumières doit être dépassé :

[U]tilitaire au plan éthique et atomisante dans sa philosophie sociale, la pensée des Lumières ne considérait la nature et la société que sous l'angle de leur signification instrumentale, ne les voyait que comme des moyens potentiels de satisfaire les désirs de l'homme. Elle espérait apporter le bonheur aux hommes grâce à une parfaite adaptation mutuelle obtenue en réorganisant les individus et la société selon des principes d'ingénierie sociale ${ }^{23}$.

Au moment où la belle totalité de la cité antique apparaît à nos yeux modernes comme chose du passé, la question est bien de retrouver une forme d'éthique sociale ou de « Sittlichkeit postindustrielle ${ }^{24}$ qui fasse droit à la liberté nouvelle de la subjectivité sans sombrer dans l'individualisme ni dans le règne de la raison instrumentale ${ }^{25}$. Telle est la raison pour laquelle Hegel reste la référence incontournable : sa philosophie permet à la fois d'éviter les illusions de la tradition utilitariste et les contre-illusions romantiques qui les suivent sans cesse.

Enfin, sur un autre registre, C. Taylor partage la critique « communautarienne » du libéralisme politique, synonyme à ses yeux d'atomisme ${ }^{26}$. Dans une perspective hégélienne là encore, il assimile le contractualisme issu de Hobbes et de Locke à une théorie instrumentalisant l'État au profit des individus de la société civile. Le philosophe québécois vise l'idéal libéral d'autonomie donné comme seule finalité légitime des institutions et la valorisation des « droitslibertés », qui sont bien plutôt des droits au retrait égoïste. Les droits de l'homme issus des Lumières seraient ceux d'un homme « sans qualités »; un tel idéal serait vide et moralement destructeur. Dans cet esprit, C. Taylor reconduit la critique hégélienne du formalisme kantien : l'autonomie ne se conçoit pas sans l'appartenance ${ }^{27}$. À l'instar d'Alisdair MacIntyre quoiqu'en un sens non réactionnaire, le philosophe critique le libéralisme individualiste issu des Lumières ${ }^{28}$. Marqué par l'héritage intellectuel d'Isaiah Berlin, il distingue les «valeurs » des Lumières (liberté, raison, primat du discours rationnel sur la violence) et leur " Projet», qui comme l'atteste l'histoire tragique du $\mathrm{XX}^{\mathrm{e}}$ siècle, peut se retourner en son contraire. Isaiah Berlin a parfaitement compris qu'il fallait aborder les Lumières par les Anti-Lumières (Counter-Enlightenment), et partir du « retournement » paradoxal du désir de liberté en réalité de l'oppression ${ }^{29}$.

Les leçons de l'histoire doivent donc être tirées : les thuriféraires des Lumières (the unruffled boosters of the Enlightenment) ont ignoré le fait qu'une valeur peut avoir besoin de son antithèse pour ne pas devenir dangereuse. La vision "panglossienne », exagérément

\footnotetext{
${ }^{23}$ C. TAYLOR, Hegel et la société moderne, op. cit., p. 69.

${ }^{24}$ Ibid., p. 124.

${ }^{25}$ Ibid., p. 132.

${ }^{26}$ C. TAYLOR, « L'atomisme », in Ph. DE LARA (dir.), La Liberté des modernes, trad. fr. Ph. de Lara, Paris, P.U.F., 1997, p. 223.

${ }^{27}$ C. TAYLOR, Hegel et la société moderne, op. cit., p. 47.

${ }^{28}$ Voir É. PERREAU-SAUSSINE, « Une spiritualité libérale ? Charles Taylor et Alisdair MacIntyre en conversation », Revue française de science politique, avril 2005, 55 (2), p. 299-315 ; D. RASMUSSEN, « Contemporary Political Theory as an Anti-Enlightenment Project », in G. Boucher, H. MArTyn Lloyd, M. Sharpe (dir.), Rethinking the Enlightenment: Between History, Philosophy, and Politics, Lanham, MD: Lexington Books, 2020, p. 39-60.

${ }^{29} \mathrm{http}$ ///berlin.wolf.ox.ac.uk/writings_on_ib/washington_tributes/taylor.html
} 
optimiste $^{30}$, omet le fait que les biens que nous valorisons peuvent être en conflit irréductible. Or négliger cette vérité peut conduire à des tragédies historiques, lorsque la réalité est sommée de correspondre à nos ambitions et à nos idéaux. Même si Taylor se démarque du libéralisme de Berlin en défendant une vision rénovée de la liberté positive ${ }^{31}$, il entérine sa thèse principale : le danger du rationalisme moderne tient à son réductionnisme moral, alors que la pluralité des valeurs demeure irréductible.

\section{Une lecture spiritualiste du politique}

Cependant, peut-on disqualifier ainsi toute une partie du «Projet des Lumières $»^{32}$ ? Cette lecture fait-elle droit aux concepts majeurs de la philosophie des Lumières dans le champ de la philosophie politique ? N'est-elle pas grevée à sa manière par une vision téléologique qui projette dans l'origine la fin tragique de l'histoire ? Ne dissimule-t-elle pas son dessein, à savoir la volonté de spiritualiser la politique et la philosophie de l'histoire?

Dans l'article de 2001 déjà cité, intitulé « The Immanent Counter-Enlightenment », C. Taylor caractérise les Lumières par le déni de la transcendance : ce «quelque chose » qui est supérieur à la vie humaine est considéré dans la philosophie des Lumières comme une illusion dangereuse, qui menace de susciter des conséquences sociales désastreuses. Le déni de toute transcendance fait naître un humanisme exclusif, fondé sur l'idée d'épanouissement humain (human flourishing) ; celui-ci ne reconnaît aucune fin légitime au-delà du bonheur de 1'humanité ${ }^{33}$. Comme l'ont souligné Quentin Skinner et d'autres, la vision religieuse de la modernité alimente ici la critique des Lumières ${ }^{34}$. C. Taylor entend saper le naturalisme éthique des Lumières pour mieux réaffirmer que le théisme est le seul véritable fondement de la morale - ce que les philosophes, Rousseau mis à part, avaient précisément exclu. Dans $A$ Catholic Modernity?, Taylor esquisse d'ailleurs un éloge du jésuite Matteo Ricci, qui voulut évangéliser la Chine ; il s'agirait de concevoir, pour notre temps, un nouveau Ricci Project ${ }^{35}$.

Certes, le philosophe nie que sa critique d'une morale sécularisée dissimule une volonté de refonder théologiquement la morale. Son ambition est plutôt de clarifier ce qui est contenu dans l'idée de « fins » de la vie morale, et de donner aux individus la motivation de les suivre grâce à la connaissance du bien constitutif (constitutive good) qui en est la source : My complaints about the more reductive strains of the Enlightenment when they shy away from constitutive good is not that they are thereby failing to 'ground' their moral stands, but (....) that they are not coming clean about their own sources ${ }^{36}$. À cet égard, la référence à Sade ne serait pas là pour montrer que l'humanisme des Lumières est infondé, mais simplement pour souligner que celui qui l'épouse (dont C. Taylor lui-même, dit-il, pour une grande part) vit sa

\footnotetext{
${ }^{30}$ Voir, pour un exemple récent, S. PINKER, Le Triomphe des Lumières, Paris, Les Arènes, 2018.

${ }^{31}$ Voir C. TAYLOR, «Qu'est-ce qui ne tourne pas rond dans la liberté négative ? (Philosophical Papers, 1979), trad. fr. Ph. De Lara, in La Liberté des modernes, op. cit., p. 255-283.

${ }^{32}$ R. WoKler, «Projecting the Enlightenment», in J. Horton, S. Mendus (dir.), After MacIntyre: Critical Perspectives on the Work of Alisdair MacIntyre, University of Notre Dame, 1994.

${ }^{33}$ C. TAYLOR, « The Immanent Counter-Enlightenment », art. cit., p. 387.

34 Quentin Skinner, «Modernity and Disenchantment: Some Historical Reflections », in J. Tully (dir.), Philosophy in an Age of Pluralism: The Philosophy of Charles Taylor in Question, Cambridge, Cambridge University Press, 1994, p. 37-48

${ }^{35}$ A Catholic Modernity?, Charles Taylor's Marianist Award Lecture, with responses by W. M. SHEA, R. LULING Haughton, G. Marsden, J. Bethke Elshtain, J. Heft, Oxford, Oxford University Press, 1999.

${ }^{36}$ C. TAYLOR, « Reply to Braybrooke and de Sousa », Canadian Review of Philosophy, 1994, 33(1), p. 125-131, ici p. 130 .
} 
vie morale en vertu de biens qui ne peuvent exister dans son ontologie propre : My plea is that we all finally put our ontologies where our (rhetorical) mouths are ${ }^{37}$.

Mais selon le philosophe de Princeton Ronald de Sousa, l'argument de C. Taylor donne lieu à une position plus fragile que celle qu'il attaque : «Car si la nature n'est pas une bonne base déductive pour la morale, tout autre fondement est entièrement arbitraire $»^{38}$. Quand bien même on accepterait la différence entre fonder la morale et en clarifier les sources, il resterait indubitable que l'alternative à la fondation naturaliste proposée par les Lumières radicales ne peut être que conventionnelle.

La généalogie de la subjectivité moderne proposée par $\mathrm{C}$. Taylor présente un autre défaut : son ancrage est moral plus que politique ; le Juste reste ici étroitement lié au Bien. Définir l'identité moderne de manière exclusivement morale paraît cependant réducteur et « unidimensionnel » - et l'on s'autorisera ici à retourner contre C. Taylor l'argument qu'il emploie contre l'hédonisme des Lumières. Dans son compte-rendu des Sources du moi, Judith Shklar n'insistait pas seulement sur l'orientation religieuse ainsi que sur la vision élitiste de la culture que propose son auteur, mais aussi sur le déficit politique dont témoigne son œuvre ${ }^{39}$. Une telle critique mérite d'être prolongée : lorsque C. Taylor juge les Lumières « radicales » contradictoires ou incapables d'assurer leurs propres fondements normatifs, il néglige semblet-il le fait que la politique des Lumières se conçoit souvent hors de tout horizon moral. Ainsi la politique conçue en faveur du peuple ou du « plus grand bien du plus grand nombre » ne reposet-elle pas nécessairement sur la justification morale de l'orientation en faveur des plus démunis. L'amélioration du sort des plus mal lotis est la finalité même d'une philosophie politique fondée sur l'égalité et la liberté originelles des hommes, qui prend en compte le fait que, comme le souligne Rousseau, il suffit de compter pour s'apercevoir que l'humanité est avant tout composée de gens du peuple : «C'est le peuple qui compose le genre humain ; ce qui n'est pas peuple est si peu de chose que ce n'est pas la peine de le compter. L'homme est le même dans tous les états : si cela est, les états les plus nombreux méritent le plus de respect $»^{40}$.

En dernière instance, la stratégie argumentative de $\mathrm{C}$. Taylor consiste à déceler ce qui est inadmissible dans la thèse adverse de son propre point de vue : la contradiction est démontrée à partir des prémisses de l'adversaire. Mais concernant les Lumières, cette stratégie ne peut être efficace qu'à la condition d'avoir au préalable opéré une double réduction de la philosophie des Lumières : réduction du naturalisme des Lumières à sa dimension matérialiste, radicale ou "utilitariste », considérée comme emblématique; réduction de la philosophie politique à la philosophie morale.

Or l'argument central de Taylor ne vaut pas dans le champ politique : la philosophie politique des Lumières avant Herder ou Kant ne postule pas, de manière générale, qu'il faut motiver le sacrifice de l'intérêt particulier à l'intérêt commun. Tout au contraire, le philosophe

\footnotetext{
${ }^{37}$ Ibid., p. 131.

${ }^{38}$ Ronald de Sousa, «Bashing the Enlightenment: A Discussion of Charles Taylor's Sources of the Self», Canadian Review of Philosophy, 1994, 33 (1), p. 109-123, ici p. 120, notre traduction.

${ }^{39}$ J. SHKLAR, « Review of Sources of the Self», Political Theory, fév. 1991, 19 (1), p. 105-109, ici p. 105.

${ }^{40}$ Rousseau, Emile, in CEuvres complètes, Paris, Gallimard, t. IV, 1969, p. 509.
} 
le plus influent du siècle, que $C$. Taylor connaît bien et qu'il cite souvent ${ }^{41}$ - Montesquieu part du présupposé diamétralement opposé : la politique moderne ne peut rien exiger de sacrificiel des individus; elle doit faire le deuil d'une politique de la vertu. Telle est la raison pour laquelle les mobiles dominants des modernes ne sont plus associés au patriotisme mais à l'amour de la liberté et au désir de reconnaissance (ce que Montesquieu nomme l'honneur et Rousseau l'amour-propre). Isaiah Berlin lui-même avait fait de Montesquieu une analyse nuancée, en l'exceptant de nombreuses critiques qu'il adressait au rationalisme des Lumières, et en reconnaissant son influence sur Herder ${ }^{42}$. En accusant une large partie de la philosophie des Lumières (surtout françaises) d'ingénuité ou de complicité avec la Terreur, Charles Taylor occulte donc tout ce qui, dans la philosophie politique pré-herderienne ou pré-kantienne, ne repose ni sur l'intérêt stricto sensu ni sur la vertu. De la même façon, Les Sources du moi minore la richesse des analyses de l'économie politique ou l'analyse sociologique du droit et des mœurs - deux voies dont la postérité sera remarquable. La vision du libéralisme contemporain censément issu des Lumières s'en trouve altérée. Le parti-pris critique de Taylor contre l'économisme et le sociologisme n'est pas anodin : à trop vouloir dénoncer les mirages du procéduralisme, les ravages de l'individualisme et les déceptions inéluctables associées à la raison instrumentale, C. Taylor a occulté la fécondité de ce qui, dans la philosophie des Lumières françaises, italiennes ou anglo-écossaises, amorce la généalogie des sciences humaines et la critique de l'économie politique.

\author{
Céline SPECTOR \\ Sorbonne Université-SND
}

\footnotetext{
${ }^{41}$ Voir par exemple C. TAYLOR, « Civil Society in the Western Tradition », in E. GROFFIER, M. PARAdIS (dir.), The Notion of Tolerance and Human Rights, Ottawa, Carleton University Press, 1991, p. 117-136.

${ }^{42}$ I. BERLIN, « Montesquieu », in À contre-courant, trad. fr. A. Berelowitch, Paris, Albin Michel, 1988, p. 200235.
} 